\title{
Braille Decoding Device Employing Microcontroller
}

\author{
Kanika Jindal, Adittee Mattoo, Bhupendra Kumar
}

\begin{abstract}
The Braille decoding method has been conventionally used by the visually challenged persons to read books etc. The designed system in the current paper has implemented a method to interface the Braille characters and English text characters. The system will help to communicate the Braille message from one visually challenged person to another as well as help us to transform the Braille language to English text through a microcontroller and a PC in order to communicate with the visually challenged persons
\end{abstract}

Keyword: Universal synchronous/Asynchronous receiver/transmitter, braille, PWM, Vibration Motor, Transistor, Diode.

\section{INTRODUCTION}

Braille mechanism was founded by Louis Braille in 1821 . Braille symbols can be construed of finger combination to 6bit binary numbers conversion. In the method discussed in this paper, individual or user wears a glove through which, switches are connected to each finger. When a particular switch to a specific finger is pressed, binary ' 1 ' will be transmitted. If the corresponding switch has been remained not pressed, it can be assumed binary logic ' 0 '. Braille characters can be assumed as a combination of 6-bit binary number with 2 columns each having 3 binary numbers shown in Fig. 1. It can be assumed as the first binary collation system of English messages. The presence and absence of switch's signal at the corresponding digits (at a, b, c, d, e, f) provides ' 1 's and ' 0 's as the binary combination [1].

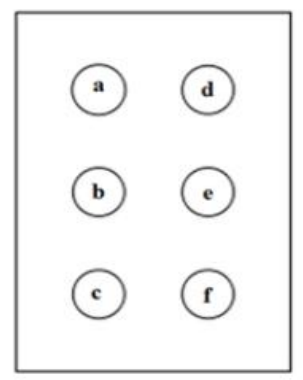

Fig. 1: Positions of binary dots in Braille system

Revised Version Manuscript Received on 10 September, 2019.

Kanika Jindal, Department of Electronics \& Communication Engineering, Noida Institute of Engineering and Technology, UttarPradesh, India.

(Email: researchnietip@gmail.com)

AditteeMattoo, Department of Computer Science \& Engineering, Noida Institute of Engineering and Technology, UttarPradesh, India.

(Email: researchnietip@gmail.com)

Bhupendra Kumar,Department of Information Technology, Noida Institute of Engineering and Technology, UttarPradesh, India.

(Email: researchnietip@gmail.com)
The proposed circuit has primary objective to recognize Braille character inputs from a visually challenged user and transmit them to another similar Braille device. This system has been embedded onto a glove that can be worn by the blind person. The first four fingers of the glove, starting from the thumb will fitted with tactile micro switches and a vibration motor. This circuit is then connected with a microcontroller and PC via RS232C cable to interface the Braille words with the $\mathrm{PC}$ based text language. The switch pressed by any person will create a Braille code that should be converted to ASCII form by ASCII conversion program for microcontroller and these letters will be seen on computer screen. This is used in order to make the characters USART compatible. Similarly, the computer text word will be reverse programmed into Braille code in order to produce vibration to respective fingers of visually challenged persons in order to make them understand the words [2]. The PC interfacing procedure is shown in Fig. 2.

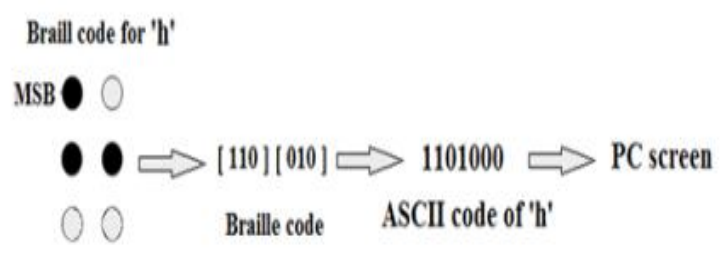

Switch closed by

finger tappings

(a)

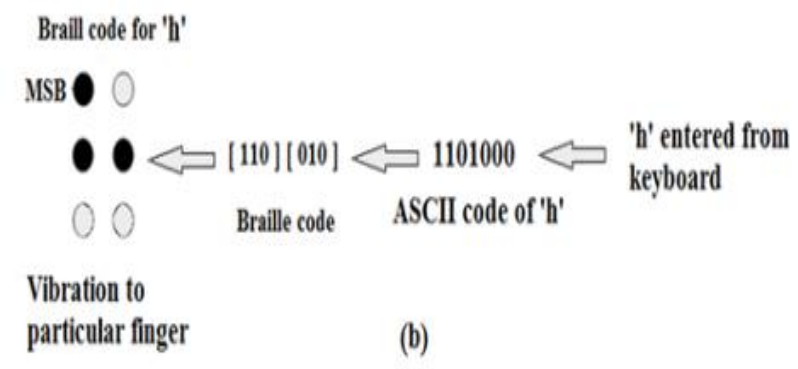

Fig. 2(a). Braille to PC communication (b) PC to Braille communication

Published By: 


\section{COMMUNICATION MODE OF OPERATION}

The designed system can work in communication mode (data to be communicated from one Braille system to another or communication between Braille and PC), store mode, record mode, read back mode and erase mode. The communication mode is most important and it combines the communication between two gloves and Braille to computer communication. For PC connections, DB-9 connector from gloves should be connected with DB-9 male port of computer. If it is linked to another glove, two DB-9 female connectors must be connected via gender changer. A hyper terminal window opens up while connecting to a PC. The necessary information like bits per second, parity bit, data bits, stop bits have to be specified. Thumb finger is used for column separator [3]. The switch information goes to Atmel Corporation's AVR ATmega16 microcontroller with proper ASCII mapping and then it is fetched to PC for display in hyper terminal. From PC, the data can be transmitted to another computer or can be stored in server.

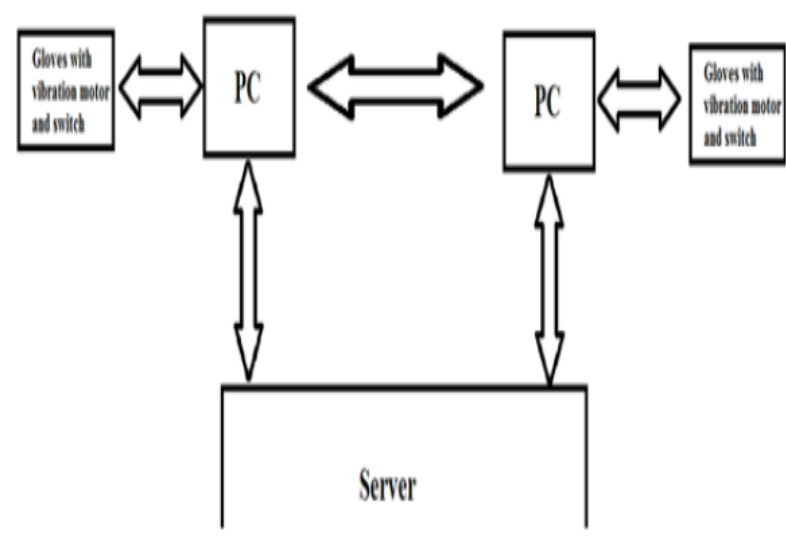

\section{Fig. 3: Communication of Braille words}

For near communication, Bluetooth, IR or ZIGBEE are normally used. In case of far remote data transfer, the data sent from Braille are stored in a file and are sent by internet with messenger protocols such as MSNP10 (MSN Messenger Protocol 10) or YMSG protocol (Yahoo Messenger protocol). It has been assumed that the total number of sender and receiver is one. A text file is auto generated in order to serve the logging of the conversation. The text file may be sent by peer-to-peer data transmission mode [4].

\section{MICROCONTROLLER CONNECTION ORIENTATION}

The AVR AT mega 16 microcontroller has the following features:

\section{RISC Architecture:}

1. 131 instruction set.

2. Single clock cycle execution.

3. 16 MIPS throughput at frequency $16 \mathrm{Mhz}$.

4. On chip 2 cycle multiplier.

5. $32 \times 8$ bit general purpose registers.
6. Nonvolatile memory.

7. $16 \mathrm{~K}$ bytes on chip programmable flash memory.

8. 512 bytes EEPROM and $1 \mathrm{~K}$ byte internal SRAM.

9. Data retention: 20 years at $85^{\circ} \mathrm{C} / 100$ years at $25^{\circ} \mathrm{C}$.

10. 10000 Flash/100000 EEPROM write cycle.

11. User programmable USART support.

12. 4 PWM channels.

The necessary circuit diagram from Braille based vibration motor and switch to microcontroller has been shown in Fig. 4.

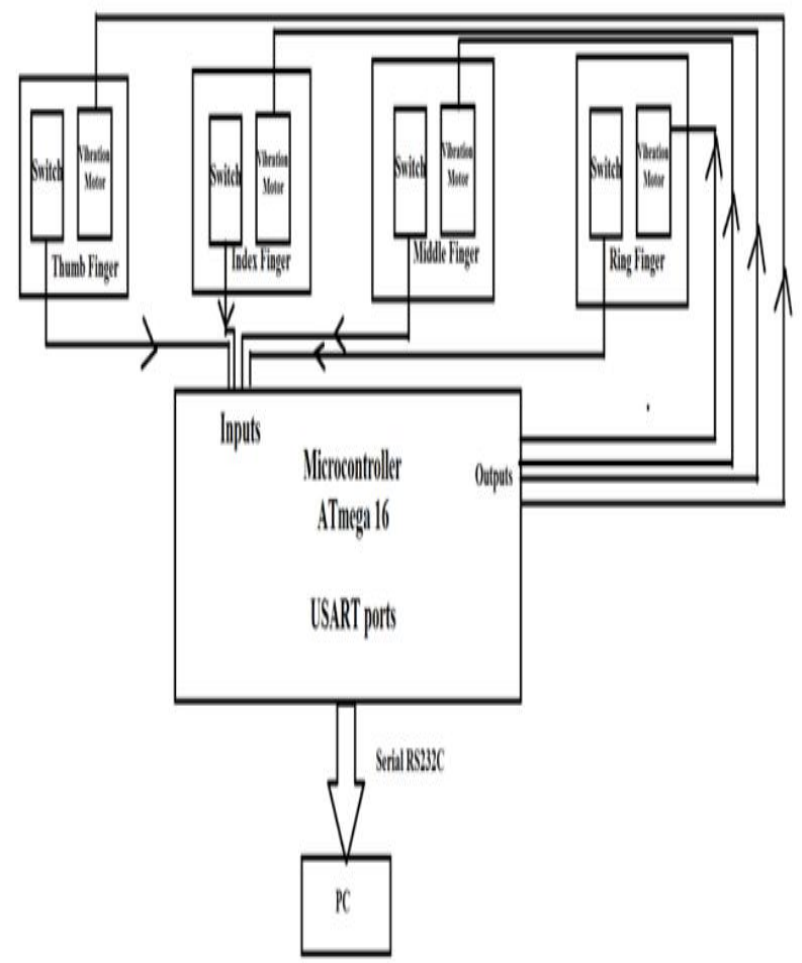

Fig., Finger connection with microcontroller and PC

For capital letters, special combination is provided in Braille language so user can distinguish any font change. The input to the microcontroller is given via switch which is connected in fingers. The switch must be pressed for at least some predetermined time interval in order to assume the number as binary ' 1 '. The following combination is then programmed to ASCII number and it is sent to PC. A level converter program has also to be written in order to make the data RS232C compatible. The Serial In-System Programming (ISP) feature in AVR ATmega16 enables us to program the microcontroller even if on runtime, it is connected to its necessary circuit. The serial programmer connection should be connected to the serial port of the computer using the DB-9 connector. The necessary hex code is downloaded in flash memory of microcontroller through the pins named MOSI and MISO. AVR Studio software changes the embedded $\mathrm{C}$ program to hex AVR code and transfer it to the microcontroller [5]. 


\section{SERIAL COMMUNICATION WITH COMPUTER}

RS 232C cable is preferably employed for serial communication b/w microcontroller and PC. For proper USART connection, RX (receiver) and Tx (transmitter) wires along with ground cables are used for this communication. For transmitting the data $\mathrm{Tx}$ is used whereas for receiving the data $\mathrm{Rx}$ is used. The necessary bits per second, number of parity bits, stop bits, frame and frequency has been decided and programmed. Correct selection of Baud rate is crucial for serial communication through $\mathrm{RS} 232 \mathrm{C}$ port. An internal remote-controlled oscillator having frequency of $8 \mathrm{MHz}$ is used as the clock circuit for our microcontroller. Baud rate is chosen of $38400 \mathrm{bps}$ for having error percentage around $0.2 \%$. UBRR value or USART Baud Rate Register is assigned to 12 for this decided setting. MAX 232C IC from Maxim Corporation is employed for converting from TTL logic to RS 232C compatible logic and vice versa. It is used as line driver/ receiver [6].

\section{VIBRATION MOTOR INTERFACING CIRCUIT\& RESULTS}

The vibration motors used in this paper is produced by LG. 12pi Coin or Flat Type Vibration Motors are preferably selected for this purpose. The snapshots are shown in Fig. 5.

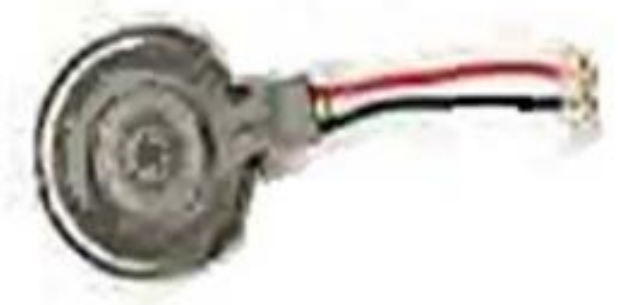

\section{Fig. 5: Snapshot of a vibration motor}

Vibration motors are attached to the individual/user fingers via gloves. The AT mega ports Port4 to Port7 are reserved for vibrational purpose for different fingers. Thumb is connected to Port4 and the ring finger is connected to Port7 pin. The operating voltage of the components is around 2.5 Volt. Maximum allowable current for safe operation is $80 \mathrm{~mA}$. The transistor BC 548 is employed to provide the enough current to propel the motors which are connected to the collector. A current limiting resistance of value $47 \Omega$ was used. The Back emf is controlled by the diode which is connected with reverse order along with vibration motors [7]. The necessary circuit diagram is shown at Fig. 6.

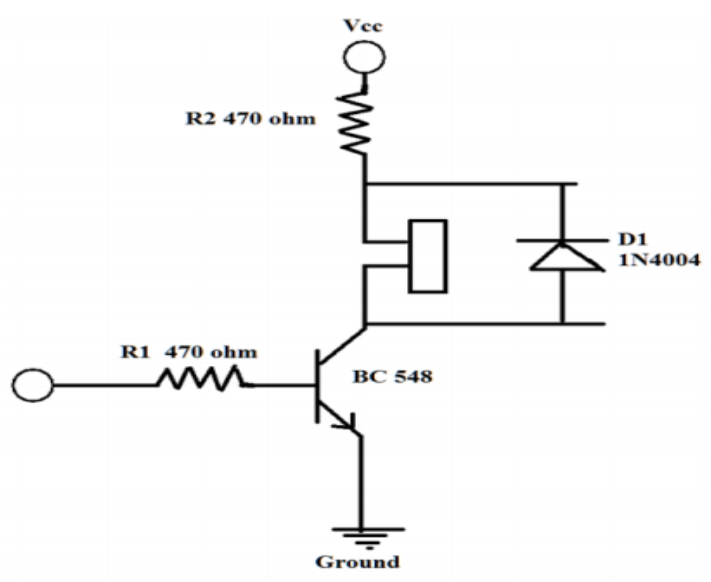

Fig. 6: Vibration motor interfacing circuit

\section{FUTURE SCOPE}

Some of the features that are desirable to be upgraded in future are mentioned below:

1. Establishment of wireless data transmission method.

2. Establishment of communication by employing USB based device because recent computers do not support DB9 connectivity.

3. Also, Lan/Internet connection method can also be employed.

\section{CONCLUSIONS}

The domain of the designed paper is documented below in summarized format

1. This system can provide direct communication b/w two blind individuals.

2. This system will also enable them to communicate with the visually challenged person via computer. The text they want to convey can be demonstrated on he computer's screen. Also, if one normal person enters some word on the $\mathrm{PC}$, then these data can be communicated via vibration motor in specified fingers.

3. The system proposed in this paper practically provides a chance to visually challenged person to use the PC by their own.

4. In future, a remote communication technique can also be employed to help visually challenged person to send their email via internet.

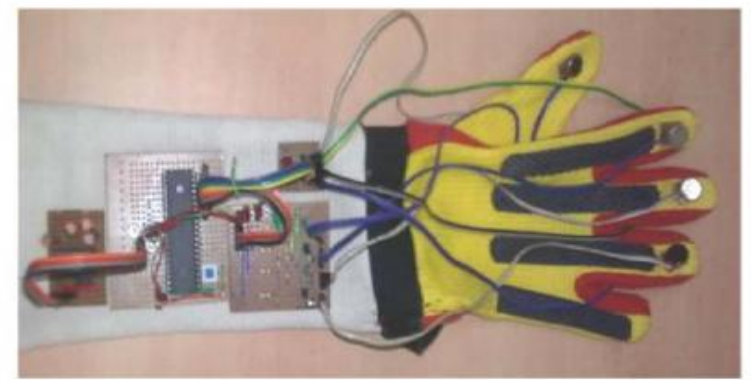

Fig. 7: Snapshot of the work with the vibration motor circuit at gloves 


\section{REFERENCES}

1. L. R. Milne, C. L. Bennett, and R. E. Ladner, "VBGhost: A Braille-based Educational Smartphone Game for Children," Proc. 15th Int. ACM SIGACCESS Conf. Comput. Access., 2013.

2. Y. Kato et al., "Sheet-type braille displays by integrating organic field-effect transistors and polymeric actuators," IEEE Trans. Electron Devices, 2007.

3. S. McCall, "Foundations of Braille Literacy. Evelyn J. Rex, Alan J. Koenig, Diane P. Wormsley \& Robert L. Baker. American Foundation For The Blind, New York, ISBN 0-89128-934-8, 153pp. US \$34.95 (Paperback)," Br. J. Vis. Impair., 2007.

4. J. B. F. van Erp, "Guidelines for the Use of Vibro-Tactile Displays in Human Computer Interaction," Proc Eurohaptics, 2002.

5. T. S. Ng, "Microcontroller," in Studies in Systems, Decision and Control, 2016.

6. L. E. Frenzel, "Controller Area Network (CAN)," in Handbook of Serial Communications Interfaces, 2015.

7. M. Sudha, S. Kirubaveni, R. Hema Latha, and S. Radha, "Design of modified power conditioning circuit for piezoelectric vibration energy harvester," in Proceedings of the 2016 IEEE International Conference on Wireless Communications, Signal Processing and Networking, WiSPNET 2016, 2016. 